\title{
DaF in der Welt: Warum es sich heute noch mehr lohnt, Deutsch zu lernen
}

\section{German as a Foreign Language: Why Learning German is worthwhile in the World today}

\begin{abstract}
Despite everything that is said and can be read currently on the news, the global amount of learners of German has not dramatically decreased in the last three decades. As a general fact, it actually has remained constant, while in some places, especially in the last few months, it has increased significantly. Nevertheless, learning German is a changing reality, its main fluctuations depending mostly on geographical and institutional factors at the educational level. In order to keep German attractive in our multilingual society, new teaching and learning concepts must be profiled, which can make foreign language acquisition suitable to a context of modern language polyphony. In any case, to learn German pays more today than ever before.
\end{abstract}

KEYWORDS: Language policy; Learning German as a foreign language.

\section{VORBEMERKUNGEN}

Der vorliegende Beitrag möchte neue Denkanregungen zum Wozu des Deutschlernens liefern, verbunden mit einigen Überlegungen nach dem Wie. Den einleitenden Rahmen bildet dabei das gegenwärtige Panorama des Deutschlernens weltweit. Im Gegensatz zu dem, was man in den Medien weiterhin noch öfters zu hören und lesen bekommt, sind die Deutschlernerzahlen auf globaler Ebene in den letzten drei Jahrzehnten keineswegs drastisch gesunken, vielmehr insgesamt konstant geblieben und mancherorts gerade in den vergangenen Monaten sogar auffällig angestiegen. Das Deutschlernen befindet sich dabei jedoch in einem Wandel, der vor allem die 
geographische wie die schulisch-institutionelle Ebene betrifft, und besonderer politischer Maßnahmen bedarf (vgl. Krumm 2006: 17-19). Um Deutsch in unserer Welt der gelebten Mehrsprachigkeit attraktiv zu gestalten, müssen Konzepte in den Vordergrund gestellt werden, die eine DaF-Aneignung sinnvoll in diesen Rahmen der modernen Sprach-Vielstimmigkeit stellen. Mögliche Gründe für die Sprachwahl des Deutschen (wie für das Erlernen jeder Fremdsprache) sind nichts statisch Festgezurrtes, sondern verändern sich laufend, eine Tatsache, die sich auch in den keineswegs starren, vielmehr beweglichen Lernerzahlen widerspiegelt, weshalb im vorliegenden Beitrag zuerst die im Wandel begriffene Situation des Deutschlernens kurz umrissen werden soll (2), um sodann unter Einbezug einer internationalen Umfrage über die Gründe für das Deutschlernen (3) eine Auflistung von sprachenpolitisch fundierten Argumenten vorzulegen (4).

\section{WELTWEITE ZAHL DER DEUTSCHLERNENDEN IM ANSTIEG}

Derzeit lernen etwa 14,5 Millionen Menschen in der Welt Deutsch. Diese Zahl wurde $2010 \mathrm{im}$ Rahmen der alle fünf Jahre stattfindenden umfassenden Untersuchung zu den Deutschlernerzahlen weltweit vom Netzwerk Deutsch erfasst (vgl. zu den internationalen DaF-Lernerzahlen auch Ammon 2010b: 102-104). In Erwartung der nächsten entsprechenden Zusammenstellung (voraussichtlich 2015) möchte ich heute durch einige punktuelle Angaben, die mir zugänglich waren, meine Begründungen für das Deutschlernen einleiten. Hier zuerst also einige Zahlen:

1. Das Goethe-Institut verzeichnet einen sehr hohen Anstieg bei seinen Sprachkursen weltweit. Die Teilnehmerzahlen sind dabei vor allem in Südeuropa gestiegen, in Spanien, Portugal, Griechenland und Italien. Nach Presseangaben haben sie sich auch in den USA, in Teilen Afrikas und in Indien erhöht. Im Vergleich zu 2011 ist die Zahl der Teilnehmer an den GI-Kursen insgesamt gesehen um gute $6 \%$ angestiegen. Zudem werden in Ländern, in denen sich politische Umbrüche vollziehen, neue Niederlassungen eröffnet, so beispielsweise 2013 in Birma, bzw. wird das Fortbildungs- und Kulturangebot ausgeweitet, wie etwa in Nordafrika - was die Zahlen künftig vermutlich noch einmal ansteigen lässt. (Goethe Institut, 6.9.2012)

2. Die Nachfrage nach Deutschkursen am Österreich Institut ergibt ein ähnlich positives Bild: von den neun international verteilten Standorten, an denen jährlich ungefähr zehntausend Menschen Deutsch lernen, meldet etwa Brno/Brünn in der Tschechischen Republik einen Zuwachs von 13\%, 
Breslau/Wroclaw ein Plus von 16\%, während sich die Zahl der Firmenkursteilnehmer in Budapest gegenüber dem Vorjahr sogar verdoppelt hat. Desweiteren treffen immer wieder Anfragen aus Ländern ein, in denen derzeit keine Österreich Institute existieren, z.B. aus China, Rumänien, der Republik Moldau, Griechenland - Anfragen, die aus budgetären Gründen gar nicht alle erfüllt werden können, trotz großem Interesse auch von Seiten Österreichs. Inhaltlich gesehen steigt dabei besonders die Nachfrage nach Fachsprachen, hier wiederum speziell nach Deutsch für Pflegeberufe (Auskünfte durch das Österreich Institut Wien; 25.02.2013).

3. Was Deutsch (genauer: DaF) an den Universitäten betrifft, wird ebenfalls von verschiedenen Seiten eine Zunahme gemeldet. So hat etwa in Italien eine landesweite Umfrage (an 30 Universitäten) durch den Italienischen Germanistenverband AIG im Dezember 2012 ergeben, dass sich die Zahl der Deutschstudenten im Vergleich zu 2011 um 37 Prozent erhöht und an einigen Universitäten Italiens (Turin, Bologna, Rom) sogar verdoppelt hat (Nied 2012). Auf schulischer Ebene sind, im selben Land, erneut hier als ein Beispiel vorgebracht, die Deutschlernerzahlen im letzten Jahr um 2,5 Prozent angestiegen (Mitteilung vom Goethe Institut Rom; 01.03.2013). Parallel zu den steigenden DaF-Lernerzahlen im Ausland erweisen sich auch die deutschsprachigen Länder selbst als attraktive Bildungsstandorte für Studierende aus dem Ausland. Im Wintersemester 2011/12 waren insgesamt 263000 ausländische Studierende an deutschen Hochschulen eingeschrieben, wobei etwa in Hessen ein Zuwachs an ausländischen Studierenden von rund 25\% zwischen 2011 und 2012 festgestellt werden konnte (vgl. Hessisches Ministerium für Wissenschaft und Kunst, http://www.hmwk.hessen. de/irj/HMWK; zuletzt abgerufen am 8.03.2013).

Diese Beispiele, die noch weiter ausgeführt werden könnten, lassen gleich im ersten Überblick erkennen, dass die gegenwärtige Erhöhung der DaF-Lernerzahlen sich vorwiegend im Rahmen der Erwachsenenbildung abspielt. Zwar lernen Hunderttausende Schüler Deutsch an Schulen in über hundert Ländern weltweit, an deutschen Auslandsschulen, an Sprachdiplomschulen oder im Unterricht anderer Schulen (erwähnt seien hier besonders auch die PASCH-Schulen, d.h. die Schulen als Partner der Zukunft, die seit 2008 ein weltweites Netzwerk von 1.530 Partnerschulen in über 110 Ländern aufgebaut haben). Die ansteigenden Zahlen der Kursteilnehmer, von denen die Medien inzwischen zu berichten beginnen (vgl. z.B. Mehlig 2012), betreffen aber überwiegend junge Menschen, die die Schule und teilweise auch schon die Hochschule abgeschlossen haben und sich auf einen Job, ein Praktikum, ein Studium - oft auch ein zweites oder weiterführendes oder eine Promotion in den deutschsprachigen Ländern vorbereiten wollen. 
Es handelt sich dabei zunehmend um gut bis sehr gut ausgebildete junge Leute, die sich durch das Deutschlernen eine Verbesserung ihrer Chancen auf dem Arbeitsmarkt erhoffen. Zahlreiche Goethe-Institute bieten entsprechend mit der vom Auswärtigen Amt geförderten Initiative "Mit Deutsch in den Beruf" unterschiedliche Fachsprachkurse an: für Ärzte, Krankenpfleger, Ingenieure, Juristen, für Berufe in der Tourismusbranche, usw.

Bis 2010 machten weltweit gesehen noch die Schüler den größten Teil an Deutschlernern aus - nur 12\% aller Deutschlerner waren im dokumentierten Zeitraum an Hochschulen oder an außeruniversitären Einrichtungen eingeschrieben (vgl. Netzwerk Deutsch, 2010: 4ff). Diese Situation kehrt sich derzeit in vielen Ländern um. In den traditionellen DaF-Lernerländern stagnieren die Zahlen oder gehen sie teilweise zurück. Dafür steigen sie allerdings in anderen Ländern an. Als Beispiel für die letzteren sei Indien genannt, wo derzeit Deutsch als erste Fremdsprache an 1.000 Schulen einer staatlichen Kette (Kendriya Vidiyalya) eingeführt wird, was bedeutet, dass in zehn Jahren ca. eine Million indischer Schulkinder Deutsch gelernt oder zumindest Grundkenntnisse darin erworben haben wird.

Das Sinken der Schülerzahlen beim Erlernen des Deutschen (meist als zweiter oder dritter Fremdsprache neben Englisch) hat zwei Hauptursachen: a) bildungspolitische Entscheidungen: Die heutige lingua franca Englisch ist in der überwiegenden Anzahl der Länder zur ersten Fremdsprache an den Schulen geworden; b) demografische Veränderungen: Sinkende Geburtenraten führen auch zu niedrigeren Schülerzahlen und damit zu sinkenden Deutschlernerzahlen. Der erkennbare positive Trend in der Erwachsenenbildung wird von den Schulleitungen und Bildungspolitikern vieler Länder leider bisher viel zu wenig wahrgenommen. Dabei müsste eine gute Schulsprachpolitik dafür sorgen, dass mindestens eine zweite Fremdsprache in den Lehrplänen verankert ist. Um hier gleich Missverständnissen vorzubeugen: Keineswegs soll gefordert werden, dass Deutsch an Stelle von Englisch unterrichtet wird. Vielmehr lautet die Devise: Erhalt der Mehrsprachigkeit (vgl. Foschi Albert 2012), gestützt auch durch die Angebote der Schulen und eine gute Schulsprachpolitik auf internationaler Ebene.

Zusammenfassend: Der Anstieg der Deutschlerner findet vor allem im Bereich der Erwachsenenbildung statt, wobei der Bildungsgrad der Interessenten zunehmend höher wird. Diese Lernergruppe scheint schon zu wissen, oder zumindest intuitiv verstanden zu haben, dass heutzutage die Anzahl der Argumente für das Deutschlernen gestiegen ist. Die folgenden beiden Abschnitte gehen der Frage nach dem "Wozu" des Deutschlernens nach, indem sie das Thema auch durch einen authentischen Blick von außen, anhand einer gerade abgeschlossenen Umfrage auf internationaler Ebene, stützen. 


\section{ERSTE ERGEBNISSE DER UMFRAGE 99 GRÜNDE DEUTSCH ZU LERNEN}

Eine vom Internationalen Deutschlehrerverband zusammen mit dem Goethe Institut, dem Österreich Institut und den Schweizer DaF-Verbänden 2012 initiierte und Ende Februar 2013 abgeschlossene Umfrage mit dem Thema 99 Gründe Deutsch zu lernen (http:/ / www.idvnetz.org) hat nicht weniger als 232 gültige Einsendungen aus 56 Ländern aller Kontinente erhalten. Aus ihrer ersten Auswertung (vgl. Hepp, im Druck) ergibt sich, dass Lerner weltweit zwar wirtschaftliche Gründe für die Sprachwahl Deutsch erwähnen, diese aber bei weitem nicht an erste Stelle setzen - eine Tatsache, auf die auch andernorts hingewiesen wird (vgl. Grucza 1995: 720; Krumm 2011: 109-110). Erhoffte wirtschaftliche Vorteile durch den Deutscherwerb werden dagegen überwiegend in Verbindung mit kulturellen Themen angegeben, bzw. mit Studien- und Weiterbildungsmöglichkeiten im deutschsprachigen Raum verflochten. Im größten Teil der Einsendungen wird das Augenmerk vor allem auf die deutschsprachige Kultur, Geschichte, Musik, Kunst, Literatur, Philosophie, Filme usw. gelegt. Die positive Erfahrung einer direkten Kontaktaufnahme mit den deutschsprachigen Regionen drückt sich dabei sehr häufig durch Beschreibung von Studienreisen, Schulpartnerschaften, Teilnahme an Tagungen und Stadtbesichtigungen aus. So wird beispielsweise die Erfahrung der Überquerung der Europabrücke von Straßburg nach Kehl als eine wertvolle Erfahrung beschrieben, durch die eine Dozentin mit einer Gruppe von Studenten in die deutsche Sprachkultur einzutauchen vermochte. $\mathrm{Zu}$ dieser direkten Kontaktaufnahme gehört auch das Besuchen von „Weltkulturerbe-Stätten“ in den deutschsprachigen Ländern - "weil das eigene Auge der König ist". Der direkte Kontakt mit den deutschsprachigen Ländern wird von vielen Einsendern unter dem Thema "Deutsch schafft Freunde" gesehen.

Interessanterweise wird in nicht wenigen Einsendungen die Struktur der deutschen Sprache in das Interessenlicht gerückt. Die Grammatik wird als "logisch strukturiert" (VR China) bzw. „systemgerecht" (Ägypten) beschrieben und gleichzeitig als "leicht" (USA) empfunden, unter der Bedingung jedoch, dass sie "richtig erklärt wird“ (Estland). Die vielen Internationalismen, besonders diejenigen lateinischer Herkunft, können „Verständnislücken schließen" (Italien). Deutsch als geeignete Sprache der Wissenschaft ermöglicht zudem, nach Meinung einiger Wettbewerbsteilnehmer/innen, ein aktiveres Mitwirken an Tagungen im deutschsprachigen Raum - was im Übrigen als Anregung betrachtet werden kann, Tagungsvorträge (weiterhin) auf Deutsch und nicht zunehmend auf Englisch zu halten. Die Lexik (,,ästhetisch anspruchsvoll mit wunderschönen Ausdrücken“ (Tunesien) und auch 
der „besondere Klang der deutschen Sprache“ (Griechenland) werden als individuelle Gründe für das Deutschlernen genannt. Die Widerspiegelung der jeweils eigenen Ausgangssprache in der Fremdsprache Deutsch ist ebenfalls ein Argument: „Deutsch ist mein Weltfenster - mit Deutsch kann ich meine eigene Sprache besser verstehen" (Brasilien).

\section{SPRACHPOLITISCHE ARGUMENTE FÜR DAS DEUTSCHLERNEN}

Aus dem sprachpolitischen Diskurs über die Vorteile des Deutschlernens für die gegenwärtige mehrsprachige Gesellschaft sind hier insgesamt acht Argumente ausgewählt worden (4.1-4.8). Sie werden jeweils abschließend durch Zitate aus der realen DaF-Welt anhand der erwähnten Umfrage untermauert.

\subsection{Englisch allein reicht nicht aus}

Auch wenn sehr gute Englischkenntnisse heute zur beruflichen Schlüsselqualifikation geworden sind, reicht die Kenntnis nur einer Fremdsprache in der heutigen globalisierten Welt bei weitem nicht mehr aus. So zielt beispielsweise auch die allgemeine Sprachenpolitik der EU darauf ab, sprachliche Vielfalt zu schützen sowie Sprachenkenntnisse zu fördern, um einerseits zur Bewahrung kultureller Identität, andererseits zu sozialer Integration beizutragen. Zum Leitprinzip der EU gehört, dass jeder Bürger zusätzlich zu seiner Muttersprache zwei Fremdsprachen beherrschen sollte (vgl. Europäische Kommission, 2012). Dabei ist selbstverständlich nicht nur EU-weit, vielmehr weltweit Mehrsprachigkeit als Voraussetzung für Verständigung und Zusammenarbeit anzusehen. Darüber hinaus bieten Fremdsprachenkenntnisse jedem Einzelnen die Möglichkeit, bildungspolitische, berufliche und wirtschaftliche Chancen zu nutzen. Ein qualitativ hochwertiges institutionelles Lehrangebot von Deutsch als Fremdsprache ist eine bildungspolitische Notwendigkeit. Diese Notwendigkeit wird grundsätzlich im Kontext von Sprachenvielfalt und Mehrsprachigkeit verstanden. Nicht Deutsch an Stelle einer (bestimmten) anderen Sprache, sondern Deutsch als Stimme im vielfältigen Chor der Sprachen soll vermittelt werden. Im Schulbereich sind demzufolge Werbe- und Lobbykampagnen besonders wichtig. Es muss aber nicht immer gleich etwas in der Größe der russlandweit angelegten „LernDeutsch!"-Kampagne sein, die (nach einem Erlass des russischen Präsidenten vom Mai 2012) die Einführung von mindestens zwei Pflichtfremdspra- 
chen an allgemeinbildenden Schulen gefördert hat (vgl. zur Politik der deutschsprachigen Länder zur Förderung der deutschen Sprache in Russland besonders Ammon, Kemper 2011). Auch das Gespräch der DeutschlehrerInnen mit den Eltern der Schüler und mit Schulleitern ist erfahrungsgemäß von großem Nutzen.

Welche weiteren Argumente für das Erlernen des Deutschen, besonders auch als zweiter und weiterer Fremdsprache, können wir zusammenstellen? Was soll der Deutschunterricht im Rahmen des vielfältigen Chors der Sprachen, im dem Englisch die erste Stimme (aber nicht die Solostimme!) einnimmt, besonders berücksichtigen?

Nach Englisch ist Deutsch weltweit die zweitwichtigste Sprache in Handel, Tourismus und Politik. Und deswegen gilt Deutsch heutzutage als die bedeutendste Fremdsprache. Wer wirklich seine Kulturkenntnisse erweitern möchte, sollte immer Deutsch als Fremdsprache auswählen. (Tunesien)

Deutsch ist heute die nach Englisch am meisten verbreitete Sprache im Internet (Bulgarien)

Gleichzeitig mit Englisch kann Deutsch die Sprache der Wissenschaft sein, im Business oder als Kommunikationsmittel dienen (Kirgistan)

Wie betrachten Deutschlerner weltweit den Gegenstand ihrer Studien, die deutsche Sprache? Gehen wir mit der Zusammenstellung an Argumenten weiter.

\subsection{Deutsch ist eine starke Welt(wirtschafts)sprache}

Deutschland, Österreich und die Schweiz gelten als wirtschaftlich starke und international geschätzte Geschäftspartner, deren wichtigste Unternehmen an fast allen bedeutenden Orten der Welt präsent sind. Viele Firmen sind dabei nicht nur im jeweiligen Inland, vielmehr auch außerhalb des Mutterlandes weltweit aktiv. Umgekehrt sind zahlreiche internationale Firmen auch in Deutschland, Österreich und der Schweiz ansässig. Damit erschlieBen Deutschkenntnisse Karrieremöglichkeiten - sowohl im eigenen Land als auch in den deutschsprachigen Ländern selbst. Zum Beispiel bestehen derzeit besondere Berufschancen in den Bereichen Biologie, Diplomatie, Finanzen, Elektrotechnik, Maschinenbau, Chemie, Pharmazie, Sport, Fahrzeugbau. Auch die Tourismusbranche bietet gute Beschäftigungsmöglichkeiten: Besucher aus Deutschland, Österreich und der Schweiz zählen in vielen Ländern zu den wichtigsten Touristengruppen. Die deutschsprachigen Länder bilden nach Ammon, Kemper (2011: 10-11) die wirtschaftlich dritt- oder 
viertgrößte Sprachgemeinschaft der Welt, hinter der englischen und der in jüngster Zeit aufgerückten chinesischen, und ungefähr gleichrangig mit der japanischen. Eine Sprache ist auch dann stark, wenn sie in mehr als einem Staat die offizielle Staatssprache ist: für Deutsch gilt das immerhin für insgesamt 7 Länder: neben Deutschland, Österreich und Liechtenstein als KoStaatssprache in der Schweiz und in Luxemburg und als regionale Amtssprache in Belgien und Italien (Südtirol). Und schließlich ist für das Interesse an einer Sprache auch wichtig, wie viele Menschen diese Sprache sprechen: Deutsch gehört, bei gut 100 Millionen Muttersprachlern, zu den 10 meistgesprochenen Muttersprachen der Welt (vgl. Lewis, 2009). Und Deutsch wird derzeit von ca. 14,5 Mio. Menschen als Fremdsprache gelernt - Tendenz steigend.

Viele Jugendliche finden eine Arbeit im eigenen Land oder im Ausland dank ihrer Deutschkenntnisse. Innerhalb Europas ist Deutsch zur Wirtschafts- und Handelssprache geworden. Der deutsche Sprachraum dient auch als Brücke, die Ost- und Westeuropa verbindet. (Spanien)

Viele meiner Studenten arbeiten schon bei multinationalen Firmen in Indien. Obwohl sie sich in anderen Hauptfächern qualifiziert haben und Deutsch nur als Nebenfach lernen, bekommen sie Stellen bei Firmen mit deutschen Kontakten. Sie verdienen sehr gut, einige sogar mehr als der Lehrer, der mit 28 jähriger Erfahrung sehr stolz auf den Erfolg seiner Studenten ist. (Indien)

\subsection{Deutsch ist eine zentrale europäische Sprache}

Die deutschsprachigen Länder liegen in der Mitte Europas und bilden das politische und wirtschaftliche Schwergewicht. Deutsch ist die stärkste Sprache in der europäischen Union, was die Sprecherzahl betrifft, es liegt hier weit vor Englisch und Französisch (vgl. Ammon, 2010 b: 93). Wer Europa kennen lernen und Kontakte in Europa aufbauen will, für den ist Deutsch, nicht zuletzt auch dank seiner nationalen Standardvarietäten, eine besonders wichtige Sprache mit großer Brückenfunktion.

In Europa sprechen über 100 Millionen Menschen Deutsch als Muttersprache, zusätzliche 80 Millionen gelten als Fremdsprachler. Deutsch ist die meistgesprochene Sprache in der europäischen Union. Lernen Sie Deutsch! (Bosnien und Herzegowina)

Mein Land, Tschechien, grenzt an zwei deutschsprachige Länder. Das verursacht die Gebundenheit an die deutsche Sprache. Deutschland ist dabei einer der stärksten Handelspartner Tschechiens. (Tschechische Republik) 


\subsection{Deutschkenntnisse bieten Möglichkeiten der Weiterbildung}

Schulen, die Deutsch als Fremdsprache anbieten, können von umfangreichen Angeboten professioneller Deutsch-Vermittler profitieren, denn die in Deutschland ansässigen Mittlerorganisationen des Auswärtigen Amts, das Goethe-Institut, der Deutsche Akademische Austauschdienst (DAAD), die Zentralstelle für das Auslandsschulwesen (ZfA), der Pädagogische Austauschdienst (PAD), das Institut für Auslandsbeziehungen (ifa) und die Deutsche Welle (DW) kümmern sich intensiv um die Realisierung des Deutsch-als-Fremdsprache-Lernens weltweit (vgl. Auswärtiges Amt, 2011). In Österreich sorgen vor allem das Bundesministerium für Unterricht, Kunst und Kultur (BMUKK), der Österreichische Austauschdienst (ÖAD), das Österreichische Sprachdiplom Deutsch (ÖSD) sowie das Österreich Institut (ÖI) für Angebote an Deutschlernende und die LehrerInnenfortbildung. Austauschprogramme: Es bestehen Abkommen zum Schüler- und Studentenaustausch zwischen den deutschsprachigen Ländern und vielen Regionen der Welt. Auf diese Weise werden jährlich tausende von Austauschprogrammen finanziert. Die deutschsprachigen Länder sind beliebte Studienorte, beispielsweise für Betriebswirtschaftslehre, Maschinenbau, Rechtswissenschaft, Medizin oder Geisteswissenschaften. Sie haben ein dichtes Bildungsnetzwerk aufgebaut und vergeben eine große Anzahl von Stipendien für Studium und Forschung.

Deutsch ist vor allem offizielle Sprache in Deutschland, Österreich, Luxemburg und in der Schweiz. Diese Länder sind in der ganzen Welt für ihre interessante und ausgezeichnete Kultur, Geschichte, Musik, Kunst und Sehenswürdigkeiten bekannt. Wer Deutsch spricht, kann in die deutschsprachigen Ländern reisen, um alles mit eigenen Augen zu sehen (weil das eigene Auge der König ist), Weltkulturerbe-Stätte besuchen und nette Menschen treffen. Es freut mich, dass sowohl in der Vergangenheit als auch heutzutage talentvolle berühmte Persönlichkeiten aus deutschsprachigen Ländern stammen. (Estland)

\subsection{Das Erlernen des Deutschen fördert interkulturelle Kompetenz}

Mehrsprachigkeit bedeutet grundsätzlich nicht nur sprachliche, sondern auch kulturelle Bereicherung. Ich möchte hier die Website des Österreichischen Außenministeriums zitieren: „Die stärkste Identifikation eines Kulturraums erfolgt weiterhin über die Sprache". Tatsächlich ist für den Aufbau dauerhafter kultureller Beziehungen zu einem Land das Erlernen der Lan- 
dessprache eine wichtige Grundlage. Vor allem: mit einer neuen Sprache lernt man immer auch verstehen, was hinter dieser Sprache steckt, die kulturellen Werte und Verhaltensweisen der Menschen. In diesem Zusammenhang wird häufig an das Bild des Eisbergs erinnert: Sprache hat eine Oberfläche, die man zuerst kennenlernt. Unter dieser Oberfläche aber transportiert sie gleichzeitig von Anfang an Werte und Wissen. Wer eine Sprache lernt, wird auch aufmerksam auf das, was unter der sprachlichen Oberfläche, der Spitze des Eisbergs, in der anderen Gesellschaft wichtig ist. Umgekehrt kann das Erlernen des Deutschen als Fremdsprache auch dazu anregen, über die Eigenheiten der eigenen Sprache, der L1, nachzudenken, sein eigenes Land und seine Mitmenschen aus einem bisher unbekannten Blickwinkel zu betrachten und über die eigenen Ansichten und Werte zu reflektieren. Dass hieraus neben einem gestärkten Bewusstsein der eigenen kulturellen Identität auch eine gewisse interkulturelle Geschicklichkeit erwachsen kann, ist gerade in der heutigen Zeit von besonderer Bedeutung. Dem Fremdsprachenunterricht fällt gerade durch die Notwendigkeit der interkulturellen Kommunikationsfähigkeit in der gegenwärtigen Welt eine besondere Rolle zu. Darüber hinaus prägt mehrsprachige institutionelle (Früh-) Erziehung die persönliche Einstellung gegenüber anderen Sprachen und Kulturen. Mehrsprachigkeit erlaubt Menschen zu verstehen, dass die Buntheit der Welt nichts Schlimmes ist, keine Angst erregen muss, sondern eine Chance darstellt. So gesehen vermag auch das Erlernen des Deutschen zur Verständigung über soziale und kulturelle Unterschiede und Grenzen hinweg beizutragen. Die interkulturelle Bildungsaufgabe des Deutschunterrichts ist damit von höchster Wichtigkeit.

Manchmal hilft das Erlernen einer Fremdsprache die eigene Sprache und Kultur besser zu verstehen durch Vergleich oder durch die Beziehung zwischen der fremden Sprache und der Muttersprache. Einige ins Ukrainisch eingewanderte deutsche Wörter kann man auch geschichtlich erklären (z.B. als Einfluss der österreichisch-ungarischen Monarchie auf die Entwicklung der ukrainischen Sprache). (Ukraine)

Ich unterrichte Deutsch an einer jüdischen Privatschule, an der es Kinder mit vielen ethnischen Hintergründen gibt (Juden, Muslime, schwarze und weiße Südafrikaner und europäische Ausländer.) Ich habe dadurch die Gelegenheit, nicht nur Sprachunterricht zu erteilen, sondern auch Landeskundliches und Völkerkundliches zu unterrichten und Vorurteile abzubauen. Die deutsche Sprache bietet dafür einen neutralen Ausgangspunkt. Da ich außerdem Englisch, Afrikaans und etwas Xhosa und Französisch spreche und ein wenig Hebräisch gelernt habe, kann ich Sprache und Kultur gut vergleichen und Unterschiede, aber auch Ähnlichkeiten hervorheben. (Südafrika) 


\subsection{Deutsch ist eine bedeutende Kultursprache}

Bis zum ersten Weltkrieg war Deutsch die Wissenschaftssprache Nummer eins. Heute gilt sie in den Geistes- und Sozialwissenschaften noch als Wissenschaftssprache Nummer zwei und liegt hier nach Ammon (2010a: $319)$ mit 7,2 \% vor Französisch (6,9\%), Russisch (2\%) und Spanisch (1,9\%). Deutsch gilt aber unbegrenzt als wichtige Kultursprache. Man denke an die folgenden Bereiche: Psychologie (Terminologie von Freud), Philosophie, Rechtswissenschaften, Musik, Literatur, teilweise auch Technik, deren Texte man gerne auf Deutsch lesen möchte.

Durch die deutsche Sprache habe ich die Möglichkeit, Bücher und Zeitungen auf Deutsch zu lesen, Filme und Theater anzusehen und damit habe ich die Möglichkeit eine andere Welt kennenzulernen und durch den "deutschen“ Himmel zu fliegen. (Brasilien)

\subsection{Deutsch ist eine ästhetisch anspruchsvolle Sprache}

Über ästhetische Werte und Wirkungen einer Sprache sind schwer objektive Urteile zu fällen. Wie Menschen in Deutschland über ihre Sprache denken, ist beispielsweise dank einer Datenerhebung des Instituts für Deutsche Sprache Mannheim aufgezeichnet worden (vgl. Gärtig, Plewnia, 2010). Daraus resultiert unter anderen, dass immerhin $75 \%$ der Befragten die deutsche Sprache "schön bis sehr schön“ finden, 43\% „anziehend“, 31\% „melodisch“. Mit anderen Worten: die deutsche Sprache ist anscheinend gar nicht so unmelodisch und hart, wie das Vorurteil oft lautet. Um es mit Dichterworten auszudrücken:

Die deutsche Sprache

ist nach allgemeinem Einverständnis

eine der wichtigsten der Welt,

tief und schwer an Sinn und Geist,

in ihren Gestalten und Bildungen unendlich

frei und beweglich, in ihren Färbungen und

Beleuchtungen der innern und äußern Welt vielseitig

und mannigfaltig. Sie hat Ton, Akzent, Musik.

Sie hat einen Reichtum, den man wirklich

unerschöpflich nennen kann und den ein

Deutscher mit dem angestrengtesten Studium

eines langen Lebens nimmer umfassen mag."

Ernst Moritz Arndt (1769-1860) 
Die Umfrage auf internationaler Ebene ergibt gerade in diesem Bereich eine hohe Anzahl an Antworten, die solch positive Wahrnehmung bestätigen, wie hier zwei aussagekräftige Beispiele belegen können:

Die deutsche Sprache ist zugleich einfach und komplex, logisch, strukturiert und trotzdem verführerisch, mit wunderschönen (nicht nur sinnvoll, sondern auch ästhetisch gesehen) Ausdrücken, rein und prächtig. (Tunesien)

Wer kann eine Kantate von Bach oder eine Oper von Mozart hören, und nicht vor Freude weinen? Oder wer kann ein Gedicht von J.W. von Goethe oder von Theodor Storm lesen, und sich von der Bildersymbolik und der Lyrik nicht beeinflussen lassen? Die Deutschen haben unser Leben durch ihre Sprache verschönert. (USA)

\subsection{Deutsch ist eine erlernbare Sprache}

Ein zweites Vorurteil, mit dem endlich aufgeräumt werden muss, lautet bekanntlich: „Deutsch ist eine schwere Sprache“. Der Schwierigkeitsgrad einer Sprache liegt nicht im Sprachsystem per se, sondern hängt von verschiedenen Faktoren ab, darunter von der Muttersprache der Lerner, d.h. von deren Verwandtschaft bzw. Ferne hinsichtlich des Deutschen als L2, wie genau so von didaktischen, situativen und individuellen (Motivation, Alter, Gender...) Gegebenheiten. So schrieb mir beispielsweise 2012 eine polnische Bachelor-Studentin - also eine Lernerin, deren Muttersprache wenige strukturelle Gemeinsamkeiten zu ihrer L2 Deutsch aufweist: „Die deutsche Grammatik ist für mich mit einem mathematischen Denkspiel vergleichbar: Beide sind logische Konstruktionen, deren Durchschauen Spaß macht.“ Und weiter: „Die Wörter und die Sätze passen wie Legosteine zueinander - dies erlaubt den Lernern von Anfang an zu kommunizieren". Deutsch wird von den Lernern selbst meist gar nicht als so schwierig empfunden, wie dies für ihre Umwelt, die Eltern oder - leider! - auch oft die Deutschlehrer selbst der Fall ist. Oder umgekehrt sind die Lernenden sogar stolz darauf, eine von ihrer Umgebung als besonders schwierig eingeschätzte Sprache zu erlernen:

Die kompositorischen Möglichkeiten der deutschen Sprache sind unerschöpflich und helfen beim Lehren, Lernen und Kulturerleben. (Russische Föderation)

Auch mache ich immer noch große Augen, wenn ich das größte deutsche Wort $\mathrm{zu}$ lesen versuche: Rindfleischetikettierungsüberwachungsaufgabenübertragungsgesetz. (Bulgarien) 
Jedes Mal, wenn ich jemandem vorgestellt werde und dazu gesagt wird, dass ich auch Deutsch spreche, kommt sofort dieser Satz zur Aussage: „Dann sind Sie aber sehr intelligent!" Alle halten Deutsch für eine schwierige Sprache. Wer Deutsch lernt und merkt, dass er/sie doch einen vollständigen, komplizierten Satz aussagen kann, hat eine doppelte Freude. (Kolumbien)

All diese Aussagen führen auch auf das „Wie“ einer angemessenen gegenwärtigen DaF-Didaktik, eine Frage, die im Rahmen der vorliegenden Ausführungen nur ansatzweise behandelt werden kann. Erwähnt werden soll an erster Stelle vor allem die Tatsache, dass heute auf weltweiter Ebene überwiegend Lernende zum Deutschunterricht kommen, für die Deutsch die zweite oder dritte Fremdsprache ist: Deutsch ist auf didaktischer Ebene gegenwärtig überwiegend eine Tertiärsprache nach der Muttersprache und der ersten Fremdsprache. Dies erfordert besondere Lehr- und Lerntechniken sowie Ansätze und Strategiefähigkeiten (im Überblick dazu Hufeisen, Marx 2010).

An zweiter Stelle ist hervorzuheben, dass eine aktuelle DaF-Didaktik auf einen durchgehenden Anspruch an Perfektion verzichten soll, wenngleich unter bestimmten Voraussetzungen und Lernanforderungen dieser Aspekt weiterhin seine Gültigkeit behält. Im Mehrsprachigkeitsansatz soll der Fokus aber vor allem auf die kommunikative Ebene gelegt werden, was gleichzeitig auch die Tatsache einbezieht, dass nicht alle Fertigkeiten auf der selben Niveaustufe angestrebt werden müssen (für manche Lernende ist beispielsweise das Leseverstehen wichtiger als das Schreiben, usw.).

Für beide dieser hier abschließend und paradigmatisch erwähnten „Wie“-Punkte gilt, dass die Stadien der Lernersprachen und die kreativen Sprachversuche im Rahmen der Mehrsprachigkeit durch positive (nicht negative) Rückmeldungen erwidert und verstärkt werden müssen. Dies schließt auch die zunehmend bewusste Berücksichtigung des Lernens als Prozess, weniger als Ziel ein. Motivationsfördernd ist hier eine positive Rückmeldung auf Kreativität, die zunehmend an die Stelle einer starren „Fehler"-Ankreidung treten sollte. Wenn etwa eine Studentin das Kompositum "Hochschuhe“ für Schuhe mit hohen Absätzen bildet (Beispiel aus Hepp 2005: 96), so hat sie damit versucht, das Wortbildungsverfahren der Komposition in der Zielsprache kreativ anzuwenden, wobei sie sich fürs erste ganz korrekt innerhalb eines vorliegenden Musters (der Zusammensetzung mit adjektivischer Bestimmungsform mit Beispielen wie Hochhaus, Hochbahn) bewegt und damit eine wichtige Stufe der Lernersprache erklommen hat.

Somit kann Deutschlernen nicht zuletzt auch mehr Spaß machen. 


\section{LITERATURVERZEICHNIS}

Ammon, U., 2010 (a). Kaum noch ein Prozent Weltanteil in den Naturwissenschaften. Über Deutsch als Wissenschaftssprache. In: Forschung und Lehre 6, 316-320.

Ammon, U., 2010 (b). Die Verbreitung des Deutschen in der Welt. In: Krumm, H.-J., Fandrych, C., Hufeisen B., Riemer, C. (Hrsg.). Deutsch als Fremd- und Zweitsprache: Ein internationales Handbuch. Berlin-New York: De Gruyter, 89-106.

Ammon, U., Kemper D. (Hrsg.), 2011. Die deutsche Sprache in Russland. Geschichte, Gegenwart, Zukunftsperspektiven. München: Iudicium.

Auswärtiges Amt (Hrsg.), 2011. Förderung der deutschen Sprache. http://www.auswaertiges-amt. de/DE/Aussenpolitik/KulturDialog/Sprache/DeutscheSprache_node.html (zuletzt abgerufen am 26.02.2013).

Außenministerium Österreich, 2013. Die stärkste Identifikation eines Kulturraums erfolgt weiterhin über die Sprache. http://www.bmeia.gv.at/aussenministerium/aussenpolitik/auslandskultur/ bildung-und-sprache.html (zuletzt abgerufen am 9.03.2013).

Gärtig, A.-K., Plewnia, A., Rothe, A., 2012. Wie Menschen in Deutschland über Sprache denken. Ergebnisse einer bundesweiten Repräsentativerhebung zu aktuellen Spracheinstellungen. Mannheim: Institut für Deutsche Sprache.

Grucza, F., 1995. Zur Geschichte und Bedeutung der deutschen Sprache in Mitteleuropa. In: Popp, H. (Hrsg.). Deutsch als Fremdsprache. An den Quellen eines Faches. Festschrift für Gerhard Helbig. (Iudicium, München), 717-727.

Europäische Kommission (Hrsg.), 2012. Sprachen 2010 und darüber hinaus. Die Rolle von Sprachen innerhalb der Lissabonstrategi. http://ec.europa.eu/languages/languages-of-europe/languages2010-and-beyond_de.htm (zuletzt abgerufen am 29.11.12).

Foschi Albert, M., 2012. Cultural Scripts and Multilingualism in the Sciences. In: Glottodidactica XXXIX/2, 7-15.

Goethe Institut (Hrsg.), 2012. Immer mehr Menschen lernen Deutsch. Pressemitteilung vom 6.9.2012. http://www.goethe.de/prs/prm/a012/de9806854.htm (zuletzt abgerufen am 8.03.2013).

Hepp, M., 2005. Fremdsprachendidaktische Aspekte der gegenwartsdeutschen Wortbildung. In: Di Meola, M., Hornung, A., Rega, L. (Hrsg.). Perspektiven eins. Akten der 1. Tagung Deutsche Sprachwissenschaft in Italien. Rom: Istituto Italiano di Studi germanici, 89-102.

Hepp, M. (im Druck). Deutsch global - Wozu heute Deutsch lernen? Die Perspektive des internationalen Netzwerks für Deutschlehrer/innen. In: Ascarelli, R. (Hrsg.). DaF-Werkstatt. Halbjahresschrift des Zentrums für die Didaktik der Deutschen Sprache an der Universität Siena-Arezzo.

Hessisches Ministerium für Wissenschaft und Kunst. Ausländische Studierende 2000-2011. http://www.hmwk.hessen.de/irj/HMWK; zuletzt abgerufen am 8.03.2013).

Hufeisen, B., Marx, N., 2010. Mehrsprachigkeitskonzepte. In: Krumm, H.-J., Fandrych, C., Hufeisen, B., Riemer, C. (Hrsg.). Deutsch als Fremd- und Zweitsprache: Ein internationales Handbuch. Berlin-New York: De Gruyter, 826-832.

Krumm, H.-J., 2006. Die Entwicklung einer österreichischen Sprachenpolitik im Kontext der europäischen Bildungs- und Sprachenprogramme. In: Faistauer, R., Cullin, I., Cali, C., Chester, K. (Hrsg.). Mehrsprachigkeit und Kommunikation in der Diplomatie. Diplomatische Akademie Wien: Favorita Papers 4, 17-34.

Krumm, H.-J., 2011. Die deutsche Sprache und die Sprachenpolitik in Europa - ein sprachenpolitischer Blick aus Deutsch als europäische Sprache. In: Cichon, P., Mitterauer, M. (Hrsg.). Europasprachen. Wien: Böhlau. 
Lewis, M.P. (Hrsg.) 2009. Ethnologue: Languages of the World. Dallas, Tex.: SIL International, 2009. http://www.ethnologue.com/ethno_docs/distribution.asp?by=size\#3 (zuletzt abgerufen am 5.03.2013).

Mehlig, H., 2012. Immer mehr lernen Deutsch. In: Süddeutsche Zeitung, 14.12.2012.

Netzwerk Deutsch (Hrsg.), 2010. Statistische Erhebungen 2010. Die deutsche Sprache in der Welt. http://www.daad.de/de/download/broschuere_netzwerk_deutsch/DeutschlernerzahlenNetzwerk_Tabelle_2010.pdf (zuletzt abgerufen am 8.03.2013).

Nied, M., 2012. Bericht Deutschstudierende an den italienischen Universitäten. http://aig.humnet. unipi.it (zuletzt abgerufen am 8.03.2013). 
